\title{
Nanogel tectonic porous gel loading biologics, nanocarriers, and cells for advanced scaffold
}

\author{
Yoshihide Hashimoto $^{\text {a,b }}$, Sada-atsu Mukai ${ }^{a, b}$, Shin-ichi Sawada ${ }^{a, b}$, Yoshihiro Sasaki ${ }^{a}$ \\ Kazunari Akiyoshi, ${ }^{\text {ab,** }}$ \\ †Department of Polymer Chemistry, Graduate School of Engineering, Kyoto University, \\ Katsura, Nishikyo-ku, Kyoto 615-8510, Japan \\ †The Exploratory Research for Advanced Technology (ERATO), Japan Science and \\ Technology Agency (JST), K’s Goban-cho Building 7, Goban-cho, Chiyoda-ku, Tokyo \\ 102-0076, Japan
}

\section{*Corresponding author}

K. Akiyoshi,

Department of Polymer Chemistry, Graduate School of Engineering, Kyoto University,

Katsura, Nishikyo-ku, Kyoto 615-8510, Japan

Tel: +81-75-383-2589, fax: +81-75-383-2590

E-mail: akiyoshi@bio.polym.kyoto-u.ac.jp 


\section{Introduction}

Polymer gels are often used as soft functional materials in biotechnological and biomedical applications [1]. For example, hydrogels are frequently used as scaffolds for tissue engineering because of their hydrated, three-dimensional (3D) structures that mimic soft biological tissues, high biocompatibility, minimal inflammatory responses, and low toxicity [2,3]. Biological activities during tissue regeneration are highly dependent on the 3D structure of the scaffold [4]. The interconnected porous structure allows rapid ingrowth of cells, promotes vascularization, and enhances nutrient diffusion or waste exchange to support successful tissue regeneration [5,6]. Porous scaffolds also provide temporary spaces and mechanical support for cells to grow, facilitating tissue regeneration [7]. Accordingly, there is a need for hydrogel scaffolds with pores of several hundred micrometers in diameter, which are large enough to allow cell ingrowth. However, the polymer network of hydrogels is generally much smaller than cells, which delays cell migration, vascularization, and tissue invasion [8]. Therefore, the design of a hydrogel scaffold with a well-controlled, microscale porous structure is a major challenge.

Recently, several strategies that combine therapeutic molecules (e.g., proteins, peptides, and growth factors) with hydrogels have been investigated to improve the performance of the scaffold. However, some of these strategies were unfavorable because proteins are often unstable and rapidly denature or degrade in the body. Therefore, novel methods are needed to handle therapeutic molecules based on hydrogels.

To address these issues in the development of hydrogel scaffolds for tissue regeneration, we investigated a novel strategy for fabricating a nanogel-based hydrogel 
scaffold with well-controlled interconnected pores that could control the release of therapeutic molecules. We first reported the development of physically cross-linked nanogels by self-assembly of associating polymers, such as amphiphilic polysaccharide. Self-assembled polysaccharide nanogels are useful components of protein carriers such as cancer and nasal vaccines [9].

More recently, a new method, termed nanogel tectonics, was proposed to construct hydrogel materials with a hierarchic structure. The nanogel-crosslinked (NanoClik) gel was synthesized using self-assembled nanogels as the building blocks [10]. Nanogels are used as individual components for building nano-integrated functional hydrogel systems. The NanoClik gel consists of a physically crosslinked network formed by self-assembly of amphiphilic polysaccharide and a chemically crosslinked network between the nanogels. The self-assembled nanogel displays chaperone-like activity (trapping proteins by hydrophobic interactions, stabilizing them, and releasing them in their native form). The chaperone activity of these nanogels is also maintained after gelation. It is possible to control the degradation and release of nanogels by the type of chemical bond responsible for the chemical crosslinks. The NanoClik gel has been used as a scaffold for tissue engineering, including bone regeneration. However, the extent of cell migration, vascularization, and tissue invasion inside the gel is limited because the gel's polymer network is very small. In this study, we developed a new hierarchic structural hydrogel with a macroporous structure using the nanogel tectonic principle. The nanogel-crosslinked porous (NanoCliP) gel with macro-sized pores of several hundred micrometers was prepared by Michael addition of acryloyl group-modified cholesterol-bearing pullulan (CHPOA) to pentaerythritol tetra (mercaptoethyl) polyoxyethylene (PEGSH), followed by freezing-induced phase 
separation. We systematically examined the structure, mechanical properties, degradation behavior, and cellular interaction of the NanoCliP gel. Finally, we subcutaneously transplanted the NanoCliP gel into mice and examined cell infiltration and neovascularization.

\section{Materials and methods}

\subsection{Chemicals}

CHP, in which pullulan (molar weight $=1 \times 10^{5} \mathrm{~g} / \mathrm{mol}$ ) was substituted with 1.2 cholesterol moieties per 100 anhydrous glucoside units and PEGSH (molar weight $=$ $1 \times 10^{4} \mathrm{~g} / \mathrm{mol}$ ) were purchased from NOF corporation (Tokyo, Japan). 2-Acryloyloxyethyl isocyanate (AOI) was purchased from Showa Denko K. K (Tokyo, Japan). Di- $n$-butyltin (IV) dilaurate (DBTDL, 95\%), Rhodamine B isothiocyanate and FITC-insulin from the bovine pancreas were purchased from Sigma-Aldrich Co., LLC (St. Louis, MO, USA). Dehydrated dimethylsulfoxide (DMSO) was purchased from Wako Pure Chemical Industries Ltd. (Osaka, Japan). Fetal bovine serum (FBS) was purchased from Life Technologies Corporation (Carlsbad, CA, USA). All other regents were obtained from commercial sources and were used without further purification.

\subsection{Synthesis of rhodamine-labeled CHP (CHP-Rh)}

CHP-Rh was synthesized as previously described [11]. Briefly, CHP (3.0 g) was dissolved in $150 \mathrm{ml}$ of dehydrated DMSO containing DBTDL (20 mM). Separately, rhodamine B isothiocyanate $(4 \mathrm{mM})$ was dissolved in dehydrated DMSO and stirred for $30 \mathrm{~min}$ at room temperature. The two solutions were combined and stirred at $45^{\circ} \mathrm{C}$ for $24 \mathrm{~h}$ and added to excess ether/ethanol. The precipitates were 
dissolved in DMSO, dialyzed with DMSO for 1 day and milli-Q water for 7 days, and then lyophilized. The degree of substitution (DS) of rhodamine groups was assessed by ultraviolet (UV) measurement at $558 \mathrm{~nm}$ using a UV/visual spectrophotometer (V-660; Jasco, Tokyo, Japan). CHP-Rh contained 0.3 rhodamine groups per 100 anhydrous glucoside units.

\subsection{Synthesis of acryloyl group-modified CHP-Rh (CHPOA-Rh)}

CHPOA-Rh was synthesized as previously described [11]. Briefly, CHP-Rh (2.5 g) was dissolved in $100 \mathrm{~mL}$ of dry DMSO, followed by the addition of DBTDL (10 $\mathrm{mM})$ and AOI (30 mM). The mixture was stirred at $45^{\circ} \mathrm{C}$ for $24 \mathrm{~h}$ and added to excess ether/ethanol. The precipitates were dissolved in DMSO, dialyzed with milli-Q water, and lyophilized. The DS of acryloyl groups was determined by ${ }^{1} \mathrm{H}$ nuclear magnetic resonance spectroscopy (400 MHz, Avance 400; Bruker biospin K.K., Kanagawa, Japan, DMSO-d6/D2O = 9/1 (v/v), 25 ${ }^{\circ} \mathrm{C}, \delta=0.64(\mathrm{~s}, 3 \mathrm{H}$, cholesterol 18-H), 0.80-2.40 (m, cholesterol H), 2.95-4.00 (glucose, 2-H, 3-H, 4-H, 5-H and 6-H), 4.69 (1H, glucose $1-\mathrm{H}(1 \rightarrow 6)), 5.03(2 \mathrm{H}$, glucose, $1-\mathrm{H}(1 \rightarrow 4)), 5.96-6.37\left(3 \mathrm{H}, \mathrm{CH}_{2}=\mathrm{CH}^{-}\right)$. The DS was 24.1 acryloyl groups per 100 anhydrous glucoside units.

\subsection{Preparation of the NanoCliP gel}

The NanoCliP gel was prepared by Michael addition of the acryloyl groups of CHPOA-Rh to the thiol groups of PEGSH. Briefly, CHPOA-Rh was dissolved in PBS ( $\mathrm{pH}$ 7.4). Separately, PEGSH was dissolved in PBS and added to the CHPOA-Rh solution. The mixture was dropped between polytetrafluoroethylene membranes and kept at $37^{\circ} \mathrm{C}$ for $1 \mathrm{~h}$ to obtain a disk-shaped nanogel-crosslinked hydrogel. The molar 


\subsection{Two-photon laser scanning microscopy (TP-LSM)}

TP-LSM of the rhodamine-labeled NanoCliP gel was conducted using a multiphoton microscope (LSM780; Carl Zeiss, Oberkochen, Germany) equipped with a Coherent Chameleon Vision S laser source and GaAsP detector. Images of the rhodamine-labeled NanoCliP gel were obtained at a magnification of $25 \times$ with an excitation wavelength of $820 \mathrm{~nm}$. A 3D image was reconstructed from a sequence of 200 fluorescent images $(1.3 \mu \mathrm{m}$ thick) acquired vertically with the $\mathrm{z}$-stack function using Zen software (Carl Zeiss).

\subsection{Freeze-fracture transmission electron microscopy (FF-TEM)}

The NanoClik and NanoCliP gels $(\varphi=4 \mathrm{~mm}$, thickness $=1.5 \mathrm{~mm})$ were immersed in $30 \%$ glycerol in PBS for 1 week at $20^{\circ} \mathrm{C}$. The specimens were frozen in slush nitrogen and fractured at $-130^{\circ} \mathrm{C}$ under a vacuum using a freeze-fracture apparatus (JFD-V; JEOL Ltd., Tokyo, Japan). The fractured surface was replicated with platinum-carbon. The replicas were washed with deionized water, mounted on copper grids, and then observed by TEM at an accelerating voltage of $100 \mathrm{kV}$ (JEM-1010; JEOL Ltd.). 


\subsection{Protein trapping and release experiments}

The NanoCliP gel $(\varphi=6 \mathrm{~mm}$, thickness $=1 \mathrm{~mm})$ was immersed in $2 \mathrm{ml}$ of FITC-insulin $(50 \mu \mathrm{g} / \mathrm{ml})$ in PBS at $25^{\circ} \mathrm{C}$. Then, $50 \mu 1$ samples of the FITC-insulin solution were obtained at specific times and the fluorescence intensity of each sample was measured using a fluorescent spectrometer (FP-8500; JASCO, Tokyo, Japan) at an excitation wavelength of $495 \mathrm{~nm}$ and an emission wavelength of $519 \mathrm{~nm}$. The 
encapsulation efficiency was estimated based on the decrease in fluorescent intensity of FITC-insulin in PBS.

The release of FITC-insulin from the NanoCliP gel in the presence of serum was also measured. The rhodamine-labeled NanoCliP gel complexed with FITC-insulin $(\varphi=6 \mathrm{~mm}$, thickness $=1 \mathrm{~mm})$ was immersed in $1 \mathrm{ml}$ of PBS supplemented with $10 \%$ FBS at $37^{\circ} \mathrm{C}$. Then, $5 \mu \mathrm{l}$ samples of the supernatant were collected at specific times and their fluorescence intensity was measured using a fluorescent spectrometer equipped with a one-drop measurement unit (SAF-851; JASCO). The experiments were repeated three times and the results are expressed as the mean \pm standard deviation (SD).

\subsection{Gel degradation experiments}

The rhodamine-labeled NanoCliP gel $(\varphi=6 \mathrm{~mm}$, thickness $=1 \mathrm{~mm})$ was immersed in $4 \mathrm{ml}$ of PBS (pH 6.02, 7.36 or 8.01 ) at $37^{\circ} \mathrm{C}$. Then, $100 \mu \mathrm{l}$ samples of PBS were collected at specific times. After collecting each sample, $100 \mu \mathrm{l}$ of fresh PBS was immediately added to maintain a fixed volume. The cumulative release of nanogels from the NanoCliP gel was estimated by fluorescent spectrometry at an excitation wavelength of $550 \mathrm{~nm}$ and an emission wavelength of $580 \mathrm{~nm}$.

We also investigated the degradation of the NanoCliP gel in the presence of serum. The rhodamine-labeled NanoCliP gel $(\varphi=6 \mathrm{~mm}$, thickness $=1 \mathrm{~mm})$ was immersed in $1 \mathrm{ml}$ of PBS supplemented with $10 \% \mathrm{FBS}$ at $37^{\circ} \mathrm{C}$. Then, $5 \mu 1$ samples of the supernatant were collected at specific times and the fluorescence intensity of each sample was measured using a fluorescent spectrometer equipped with a one-drop measurement unit. The experiments were repeated four times, and the results are expressed as the mean $\pm \mathrm{SD}$. 


\subsection{Preparation of liposomes}

Nitrobenzoxadiazole (NBD)-labeled liposomes were prepared using conventional methods [11]. DOPC and NBD-labeled phosphatidylcholine (PC) were dissolved in chloroform at a molar ratio of 1000:1 and were gently evaporated by rotating under a stream of argon gas. The lipid film was dried overnight in a vacuum. The resulting lipid film was hydrated with PBS (pH 7.4) for $1 \mathrm{~h}$ at $50^{\circ} \mathrm{C}$ and vortexed lightly to remove any remaining lipids from the glass tube. The NBD-labeled DOPC liposomes were prepared by sequential extrusion of the obtained vesicles through polycarbonate membranes at $50^{\circ} \mathrm{C}$. The mean diameter of the liposomes was measured by dynamic light scattering.

\subsection{Use of the NanoCliP gel as a liposome reservoir}

The rhodamine-labeled NanoCliP gel $(\varphi=6 \mathrm{~mm}$, thickness $=1 \mathrm{~mm})$ was immersed in $400 \mu \mathrm{l}$ of NBD-labeled DOPC liposomes $(3 \mu \mathrm{M})$ in PBS for $24 \mathrm{~h}$ at $4{ }^{\circ} \mathrm{C}$. The resulting complex was washed with PBS to remove excess liposomes. Images of the complex were taken using a multi-photon microscope (LSM780; Carl Zeiss) at a magnification of $10 \times$ with an excitation wavelength of 488 and emission wavelength of $561 \mathrm{~nm}$.

\subsection{Cell culture and cell morphology}

Mouse embryonic fibroblasts (NIH3T3, clone 5611; JCRB cell bank, Osaka, Japan) were maintained in Dulbecco's modified Eagle's medium (DMEM; Gibco/Life Technologies Corporation) supplemented with $10 \%$ FBS and $1 \%$ antibiotics. The 
NanoCliP gel $(\varphi=6 \mathrm{~mm}$, thickness $=1.5 \mathrm{~mm})$ was coated with fibronectin derived from rat plasma (50 $\mu \mathrm{g} / \mathrm{gel}$; Calbiochem/EMD Millipore, Billerica, MA, USA) before cell seeding and was placed in PBS for $24 \mathrm{~h}$ to remove excess fibronectin. Cells were detached with $0.25 \%$ trypsin/ $1 \mathrm{mM}$ tetrasodium ethylene diamine tetraacetic acid (Life Technologies Corporation) and were counted by the trypan blue exclusion method. Cells were seeded onto the NanoCliP gel over an area of $0.28 \mathrm{~cm}^{2}$ at a density of $1.5 \times$ $10^{4} \mathrm{cells} / \mathrm{cm}^{2}$, and were maintained in serum-free DMEM in a humidified atmosphere of $95 \%$ air $/ 5 \% \mathrm{CO}_{2}$ at $37^{\circ} \mathrm{C}$ for $24 \mathrm{~h}$. After being washed two times, the cells were stained with calcein-AM and propidium iodide (Cellstain Double Staining kit; Dojindo Laboratories, Kumamoto, Japan), and were observed by LSM (LSM780, Carl Zeiss).

\subsection{Subcutaneous transplantation}

Three female BALB/c mice aged 6 weeks (20 g) were purchased from Japan SLC Co. (Shizuoka, Japan). All animals were treated in accordance with the guidelines of the National Institutes for Health Animal Health and Use Committee. All animal experiments were approved by the ethics committee for animal welfare of Kyoto University. Mice were anesthetized with 3 vol\% isoflurane (Mylan Inc., Cecil Township, PA, USA), and anesthesia was maintained with $1.5 \%$ isoflurane. The dorsal skin was shaved, disinfected with iodine and $70 \%$ ethanol, and then two small subcutaneous pockets were made on each side of the dorsal column. Samples of the NanoCliP gel $(\varphi=6 \mathrm{~mm}$, thickness $=1 \mathrm{~mm})$ were gently inserted into the pockets using a spatula and the pockets were closed using 5-0 nylon sutures. Four weeks later, the mice were sacrificed with an overdose of pentobarbital sodium (Somnopentyl; Kyoritsu 
Seiyaku Corporation, Tokyo, Japan). The dermis, including the transplanted NanoClip gel, was excised for H\&E staining and immunohistochemistry.

\subsection{Histological analysis}

The specimens were fixed in $4 \%$ paraformaldehyde at room temperature for $24 \mathrm{~h}$, washed, and immersed in a stepwise manner in 10-30\% sucrose in PBS followed by OCT compounds. The specimens were rapidly frozen in liquid nitrogen and were then cryosectioned at a thickness of $10 \mu \mathrm{m}$ using a cryostat (Leica CM1850, Leica Microsystems GmbH, Wetzlar, Germany). The sections were stained with hematoxylin and eosin (H\&E; Leica Surgipath SelecTech system). For immunohistochemistry, the sections were treated with $3 \%$ hydrogen peroxide in PBS to inactivate endogenous peroxidase, and were incubated with rat anti-mouse F4/80 antibody (1:50 dilution, clone CI:A3-1; AbD Serotec, Kidlington, Oxfordshire, UK) overnight at $4^{\circ} \mathrm{C}$. After being washed in PBS, the sections were incubated with horseradish peroxidase-conjugated goat anti-rat immunoglobulin $\mathrm{G}$ antibody (1:200 dilution, Life Technologies Corporation) for $1 \mathrm{~h}$ at room temperature. The sections were then washed, treated with 3,3'-diaminobensidinetetrahydrochloride, and counterstained with hematoxylin. The sections were observed under a fluorescence microscope equipped with a digital camera (BIOREVO BZ-9000; Keyence, Tokyo, Japan).

\subsection{Statistical analysis}

All results are expressed as the mean \pm SD. Analysis of variance followed by Student's $t$ test was used to detect significant differences among the experimental groups. Values of $P<0.05$ were considered statistically significant. 


\section{Results and discussion}

\subsection{Preparation of polysaccharide-PEG crosslinked porous gels}

CHPOA formed a nanogel ( $~ 50 \mathrm{~nm}$ in diameter) by self-assembly in water.

The NanoClik gel was prepared by Michael addition of the acryloyl groups of a nanogel and thiol groups to PEGSH (Fig. 1). Hydrogels were also developed using acryloyl group-modified pullulan (POA) (pullulan-crosslinked gel, PClik gel) in a similar manner. Gelation was monitored using a shear rheometer at a fixed angular frequency $(\omega=2 \pi \mathrm{rad} / \mathrm{s})$ and strain amplitude $(\gamma=0.5 \%)$. The typical gelation kinetics (storage modulus [G'] and loss modulus [G"]) of 2\% CHPOA nanogel and POA solutions at a crosslink concentration of $3.6 \mathrm{vol} \%$ (acryloyl:thiol $=2: 1$ molar ratio) were measured as a function of the reaction time (Fig. S1). G' increased rapidly between 100 and $500 \mathrm{~s}$, and then continued to increase over time but at a slower rate. The gelation behavior did not differ between these hydrogels.

Porous gels can be prepared by freeze-drying (i.e., lyophilization), porogen leaching, gas foaming, emulsion templating, and electrospinning, for example [13]. In this study, we prepared the NanoCliP gel by freezing-induced ice-polymer phase separation. The disk-shaped NanoClik and PClik gels were transferred to a polystyrene dish, and the water inside the hydrogel was frozen at $-28^{\circ} \mathrm{C}$ for $30 \mathrm{~min}$ and then thawed at $25^{\circ} \mathrm{C}$. Visualizing the morphology of hydrogels in their native state is very important when assessing the properties of hydrogels. Scanning electron microscopy (SEM) is often used for the structural analysis of hydrogels in biomedical fields. However, because this procedure requires the removal of water before observation, it cannot be used to assess the native state of hydrogels, which should be characterized in the 
presence of water. Meanwhile, there are currently no methods capable of determining the porosity, pore size, or structure of soft and highly hydrated materials [14]. TP-LSM has become widely used in biomedical research because it yields high-resolution images of thick biological specimens with superior contrast to other imaging modalities [15]. Accordingly, we used TP-LSM to assess the structure of the gels in their hydrated state.

TP-LSM imaging revealed that the NanoClik gel had a homogeneous structure (Fig. S2a), although SEM indicated that the gel had a porous structure (Fig. S2b). TP-LSM imaging revealed the true structure of the hydrogel in water. Freezing-induced phase separation of the NanoClik gel yielded a relatively uniform porous structure with a pore diameter of several hundred micrometers (Fig. 2a). These pores were interconnected deep below the gel's surface (Fig. 2b). The porosity of the NanoCliP gel estimated from the analysis of multiple 3D images was about $84 \%$. By contrast, the pores in the PClik gel subjected to the same treatment were separated by an irregular thick polymer phase (Fig. 2c), and the pore interconnections were sparsely distributed (Fig. 2d). The porosity of the pullulan-crosslinked porous (PCliP) gel was about $47 \%$. The phase separation between water (ice) and the polymer differed between the nanogel structure (NanoClik gel) and the random conformation of the pullulan chain (PClik gel) in the gel network. The NanoClik gel enhanced phase separation and formed a well-developed interconnected porous structure. Ice crystallization occurs because of the nucleation of an ice embryo in the supercooled state, and is followed by crystal growth [16]. Some substances activate or inhibit ice nucleation $[17,18]$. The rate of ice crystal growth depends on the density of the non-ice phase between ice crystals because water molecules must diffuse in the non-ice phase to yield coarse ice crystals. Cholesterol is an ice nucleator that disrupts the supercooled state [19]. Therefore, the 
structural differences between the NanoCliP and PCliP are derived from the features of each nanogel, especially the presence of cholesteryl domains, which act as physical crosslinking points.

The structure of the nanogels before and after freezing-induced phase separation was investigated by FF-TEM. Figure 3a and b show the typical FF-TEM images of the NanoClik and NanoCliP gel, respectively. The nanodomains formed by the CHPOA nanogels were observed in the NanoClik gels. By contrast, the FF-TEM image of the NanoCliP gels revealed that the nanodomains are densely packed and the nanogel's structure is well maintained, indicating that freezing did not affect the nanogels.

\subsection{Characteristics of crosslinked nanogels}

Figure $4 \mathrm{a}$ shows the results of the time-dependent dynamic viscoelastic analyses of the NanoCliP gel. Interestingly, both G' and G" of the NanoCliP gel were about 10 times higher than those of the NanoClik gel, whereas G' of the pullulan-crosslinked porous (PCliP) gel was about $50 \%$ of that of the pullulan-crosslinked (PClik) gel (Fig. 4b). The storage modulus G' for the NanoCliP gel was similar to that of chemically crosslinked gels, such as polyacrylamide [20], and physically crosslinked polysaccharide gels, such as agarose [21]. Once the ice crystals grow larger than the polymer network's mesh, the fixed polymer network becomes concentrated near the surface of the ice crystals. Consequently, the local concentrations of CHPOA and PEGSH increased in the frozen-concentrated phase, allowing additional crosslinking reactions, which might increase the viscoelastic modulus. Furthermore, the 


\subsection{Interactions between the NanoCliP gel and Proteins}

CHP nanogels can interact with proteins and encapsulate them within the nano-matrix $[25,26]$. The encapsulation of FITC-insulin as a model protein in the NanoCliP gel was monitored by the decrease in the fluorescent intensity of the FITC-insulin solution. Figure 5a shows the time-course of the interactions between the 
hydrogel and FITC-insulin. The amount of FITC-insulin trapped in the hydrogel gradually increased over time, reaching an equilibrium within $48 \mathrm{~h}$. More than $80 \%$ of the FITC-insulin was spontaneously trapped in the gel. Fluorescence microscopic observation showed that FITC-insulin interacted with the NanoCliP gel in a uniform manner (Fig. S3). CHP nanogels mainly trap proteins via hydrophobic interactions. Therefore, insulin probably binds to the CHPOA nanogel building blocks in the NanoCliP gel. We previously reported that proteins encapsulated in nanogels are released in the presence of excess serum albumin via an exchange reaction [27]. Figure $5 \mathrm{~b}$ shows the release of FITC-insulin from the NanoCliP gel in the presence of serum. FITC-insulin was not rapidly released from the gels in a burst, rather, FITC-insulin was released in a controlled and sustained manner. We also investigated the degradation of the gels in the presence or absence of serum (Fig. S4). The NanoCliP gel was formed by crosslinks between the acryloyl group of CHPOA and the thiol group of PEGSH via ester bonds. Therefore, the nanogels can be gradually released from the NanoCliP gel

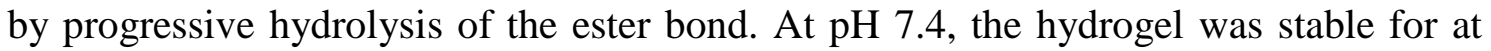
least 40 days in the absence of serum. However, in the presence of serum, the gel degraded within 20 days. This is because serum contains several esterases. During the insulin-release experiment, we observed almost no degradation of the NanoCliP gel, even though $\geq 90 \%$ of the insulin was released. For many gel-protein complexes, the protein is released as a burst during the early phase because proteins encapsulated in hydrogels are released by diffusion through the polymer network. By contrast, FITC-insulin is stably encapsulated within the nanogel distributed throughout the NanoCliP gel. Therefore, the release of insulin from the gel is probably due to an exchange of insulin for serum albumin. 


\subsection{Using the NanoCliP gel as a liposome reservoir}

CHP nanogels can form a complex with liposomes because the cholesteryl groups of CHP form anchors in the lipid bilayer of liposomes. The liposome-nanogel material is an interesting dual nanocarrier delivery system or multidrug delivery system, allowing the sequential release of the nanogels and liposomes [28]. Therefore, we investigated the interaction between the NanoCliP gel and DOPC-liposomes $(\sim 100 \mathrm{~nm}$ in diameter). Figure 6 shows the confocal images of the NanoCliP gel complexed to DOPC-liposomes. The DOPC-liposomes interacted with the NanoCliP gel and homogeneously penetrated into the gel matrix. These complexes were stable for $\geq 3$ weeks in PBS (pH 7.4) at $4^{\circ} \mathrm{C}$.

\subsection{Cell culture on the NanoCliP gel}

Extracellular matrix (ECM) proteins such as laminin, fibronectin, and vitronectin, which enhance cell adhesion, do not readily absorb to the gel surface because of the hydrophilic properties of hydrogels [29,30]. Indeed, poly(ethylene glycol) (PEG) hydrogels and polysaccharide-based hydrogels like agarose are very resistant to protein adsorption and cell adhesion [31]. However, because the NanoCliP gel contained nano-sized hydrophobic domains, it may be able to adsorb ECM proteins on its surface. To confirm this possibility, we first investigated the interaction between the NanoCliP gel and fibronectin. Fluorescence microscopic observation revealed that the NanoCliP gel readily interacted with fibronectin, and this interaction was maintained for $\geq 3$ weeks, even after washing the gel (Fig. S5). 
To assess whether cells could adhere to the fibronectin-coated NanoCliP gel, we conducted a live-dead assay $24 \mathrm{~h}$ after seeding mouse embryonic fibroblasts (NIH3T3) onto the gel. In the live-dead assay, living cells appear green (Fig. 7a) and dead cells appear red (Fig. 7b). After seeding fibroblasts onto the NanoCliP gel, many of the cells passed through the interconnected macropores of the NanoCliP gel before adhering to the gel, while some cells adhered in a round colony and spread out along the surface of the NanoCliP gel (Fig. 7c,d). Additionally, some cells penetrated the NanoCliP gel to a depth of approximately $80 \mu \mathrm{m}$ without triggering cell death (Fig. 7e,f). The magnified inset in Figure $7 \mathrm{e}$ shows that the adhered cells were elongated. These results indicate that the interconnected macropores of the NanoCliP gel allowed the cells to penetrate deeper into the gel, which may facilitate tissue remodeling in wound healing.

\subsection{Subcutaneous transplantation}

Recently developed tissue engineering strategies use scaffolds with a porous structure that contain exogenous growth factors to stimulate or augment tissue repair [32,33]. Several hydrogels have been investigated as possible 3D scaffolds for use in tissue engineering [34,35]. However, many cells types cannot adhere to commonly used hydrogels, except for natural ECM proteins like collagen [32]. Additionally, cell adhesion proteins such as fibronectin, which binds to integrin receptors located in the cell surface plasma membrane, do not adsorb to the hydrophilic surface of hydrogels [30,36]. To show whether the porous, interconnected structure of the NanoCliP gel remained intact in vivo and allowed cell infiltration and neovascularization, the NanoCliP gels were subcutaneously transplanted into mice. Four weeks after 


\section{Conclusions}

We have developed a new multifunctional, porous amphiphilic gel (the NanoCliP gel) by a combination of a simple template strategy and nanogel tectonics. The gel possessed relatively strong mechanical properties, biodegradability, and was capable of sustained protein release. The NanoCliP gel was also able to trap proteins, liposomes, and cells on the surface of the porous domain. The NanoCliP gel also 
stimulated cell infiltration, tissue ingrowth, and neovascularization in the absence of exogenous growth factors. Taken together, the present results suggest that the NanoCliP gel represents a new universal platform as a scaffold for tissue engineering.

\section{Acknowledgements}

We wish to thank K. Umesaki and S. Ikeda of Kyoto University for preparing the liposomes. This work was supported by the Exploratory Research for Advanced Technology of the Japan Science and Technology Agency (JST-ERATO). 


\section{References}

[1] Saul JM, Williams DF. Hydrogels in regenerative medicine. Handbook of polymer application in medicine and medical devices, 2013; 279-301.

[2] Madden LR, Mortisen DJ, Sussman EM, Dupras SK, Fugate JA, Cuy JL, et al. Proangiogenic scaffolds as functional templates for cardiac tissue engineering. Proc Natl Acad Sci USA 2010; 107(34): 15211-6.

[3] Van Vlierberghe S, Dubruel P, Schacht E. Biopolymer-based hydrogels as scaffolds for tissue engineering applications: a review. Biomacromolecules 2011; 12(5): $1387-408$.

[4] Oh SH, Park IK, Kim JM, Lee JH. In vitro and in vivo characteristics of PCL scaffolds with pore size gradient fabricated by a centrifugation method. Biomaterials 2007; 28(9): 1664-71.

[5] Hollister SJ. Porous scaffold design for tissue engineering. Nat Mater 2005; 4: $518-24$.

[6] Murphy CM, Haugh MG, O'Brien FJ. The effect of mean pore size on cell attachment, proliferation and migration in collagen-glycosaminoglycan scaffolds for bone tissue engineering. Biomaterials 2010; 31(3): 461-6.

[7] Eiselt P, Yeh J, Latvala RK, Shea LD, Mooney DJ. Porous carriers for biomedical applications based on alginate hydrogels. Biomaterials 2000; 21(19): 1921-7.

[8] Zisch AH, Lutolf MP, Ehrbar M, Raeber GP, Rizzi SC, Davies N, et al. Cell-demanded release of VEGF from synthetic, biointeractive cell-ingrowth matrices for vascularized tissue growth. FASEB J 2003; 17(15): 2260-2. 
[9] Sasaki Y, Akiyoshi K. Nanogel engineering for new nanobiomaterials: from chaperoning engineering to biomedical applications. Chem Rec 2010; 10(6): $366-76$.

[10] Shimoda A, Yamamoto Y, Sawada S, Akiyoshi K. Biodegradable nanogel-integrated hydrogels for sustained protein delivery. Macromol Res 2012; 20(3): $266-70$.

[11] Ayame H, Morimoto N, Akiyoshi K. Self-assembled cationic nanogels for intracellular protein delivery. Bioconjugate Chem 2008; 19(4): 882-90.

[12] Samad A, Sultana Y, Aqil M. Liposomal drug delivery systems: an update review. Curr Drug Deliv 2007; 4(4): 297-305.

[13] Annabi N, Nichol JW, Zhong X, Ji C, Koshy S, Khademhosseini A, et al. Controlling the porosity and microarchitecture of hydrogels for tissue engineering. Tissue Eng Part B 2010; 16(4): 371-83.

[14] Plieva F, Huiting X, Galaev IY, Bergenstahl B, Mattiasson B. Macroporous elastic polyacrylamide gels prepared at subzero temperatures: control of porous structure. J Mater Chem 2006; 16(41): 4065-73.

[15] Helmchen F, Denk W. Deep tissue two-photon microscopy. Nat Methods 2005; 2: $932-40$.

[16] Hobbs PV. Ice Physics, Clarendon Press, Oxford, UK 1974.

[17] Raymond JA, DeVries AL. Adsorption inhibition as a mechanism of freezing resistance in polar fishes. Proc Natl Acad Sci USA 1977; 74(6): 2589-93.

[18] Raymond JA, Wilson P, DeVries AL. Inhibition of growth of nonbasal planes in ice by fish antifreezes. Proc Natl Acad Sci USA 1989; 86(3): 881-5. 
[19] Cochet N, Widehem P. Ice crystallization by Pseudomonas syringae. Appl Microbiol Biotechnol 2000; 54(2): 153-61.

[20] Calvet D, Wong JY, Giasson S. Rheological monitoring of polyacrylamide gelation: Importance of cross-link density and temperature. Macromolecules 2004; 37(20): 7762-71.

[21] Normand V, Lootens DL, Amici E, Plucknett KP, Aymard, P. New insight into agarose gel mechanical properties. Biomacromolecules 2000; 1(4): 730-8.

[22] Abdurrahmanoglu S, Can V, Okay O. Design of high-toughness polyacrylamide hydrogels by hydrophobic modification. Polymer 2009; 50(23): 5449-5455.

[23] Ikeda S, Nishinari K. "Weak gel"-type rheological properties of aqueous dispersions of nonaggregated kappa-Carrageenan helices. J Agric Food Chem 2001; 49(9): 4436-41.

[24] Ross-Murphy SB, Morris VJ, Morris ER. Molecular viscoelasticity of xanthan polysaccharide. Faraday Symp Chem Soc 1983; 18: 115-29.

[25] Nomura Y, Ikeda M, Yamaguchi N, Aoyama Y, Akiyoshi K. Protein refolding assisted by self-assembled nanogels as novel artificial molecular chaperone. FEBS Lett 2003; 553(3): 271-6.

[26] Nochi T, Yuki Y, Takahashi H, Sawada S, Mejima M, Kohda T, et al. Nanogel antigenic protein-delivery system for adjuvant-free intranasal vaccines. Nat Mater 2010; 9: 572-8.

[27] Akiyoshi K, Kobayashi S, Shichibe S, Mix D, Baudys M, Kim SW, et al. Self-assembled hydrogel nanoparticle of cholesterol-bearing pullulan as a carrier of protein drugs: Complexation and stabilization of insulin. J Control Release 1998; 54(3): 313-20. 
[28] Sekine Y, Moritani Y, Ikeda-Fukazawa T, Sasaki Y, Akiyoshi K. A hybrid hydrogel biomaterial by nanogel engineering: bottom-up design with nanogel and liposome building blocks to develop a multidrug delivery system. Adv Healthc Mater 2012; 1(6): 722-8.

[29] Atala A, Mooney DJ. Synthetic Biodegradable Polymer Scaffolds. Springer, 1997.

[30] Drury JL, Mooney DJ. Hydrogels for tissue engineering: scaffold design variables and applications. Biomaterials 2003; 24(24): 4337-4351.

[31] Hou Y, Schoener CA, Regan KR, Munoz-Pinto D, Hahn MS, Grunlan MA. Photo-cross-linked PDMSstar-PEG hydrogels: synthesis, characterization, and potential application for tissue engineering scaffolds. Biomacromolecules 2010; 11(3): $648-56$.

[32] Mariner PD, Wudel JM, Miller DE, Genova EE, Streubel SO, Anseth KS. Synthetic hydrogel scaffold is an effective vehicle for delivery of INFUSE (rhBMP2) to critical-sized calvaria bone defects in rats. J Orthop Res 2013; 31(3): 401-6.

[33] Kearney CJ, Mooney DJ. Macroscale delivery systems for molecular and cellular payloads. Nat Mater 2013; 12: 1004-17.

[34] Rice JJ, Martino MM, Laporte LD, Tortelli F, Briquez PS, Hubbell JA. Engineering the regenerative microenvironment with biomaterials. Adv Healthc Mater 2013; 2(1): 57-71.

[35] Jiang Y, Chen J, Deng C, Suuronen EJ, Zhong Z. Click hydrogels, microgels and nanogels: Emerging platforms for drug delivery and tissue engineering. Biomaterials 2014; 35(18): 4969-85. 
1
[36] Jeong SI, Krebs MD, Bonino CA, Khan SA, Alsberg E. Electrospun alginate nanofibers with controlled cell adhesion for tissue engineering. Macromol Biosci 2010; 10(8): 934-43. 


\section{Figure legends}

Figure 1 | Michael addition of CHPOA nanogel to PEGSH.

Figure 2 | Pseudo-coloured TP-LSM images of the rhodamine-labeled NanoCliP and PCliP gels. (a,c) Two-photon 3D images from the surface of the NanoCliP (a) and PCliP (c) gels to a depth of $400 \mu \mathrm{m}$. (c,d) Cross-sectional two-photon 3D images of the NanoCliP (b) and PCliP (d) gels. Scale bar $=100 \mu \mathrm{m}$.

Figure 3 | FF-TEM images of the NanoClik (a) and NanoCliP (b) gels. Scale bar = $200 \mathrm{~nm}$.

Figure 4 | Storage modulus G' $(\diamond)$ and loss modulus G' $(\triangle)$ of each gel. $(a, b)$ Time-dependent $(\omega=1 \mathrm{rad} / \mathrm{s}, \gamma=0.5 \%)$ and $(\mathrm{c}, \mathrm{d})$ dynamic frequency-sweep $(\omega=$ $10^{-1}-10^{2} \mathrm{rad} / \mathrm{s}, \gamma=0.5 \%$ ) measurements of the NanoClik and PClik gels before and after freezing-induced phase separation. (e-g) Quantitative analysis of G' (e), G” (f) and the loss factor $\tan \delta(\mathrm{g})$. Results are expressed as the mean \pm standard deviation $(\mathrm{n}=4)$. $* \mathrm{p}<0.05, * * \mathrm{p}<0.005$, and ***p $<0.0001$ (two-tailed Student's $t$ test).

Figure 5 | Protein encapsulation and release profiles of the NanoCliP gel. (a) Encapsulation efficiency of FITC-insulin by the NanoCliP gel. (b) Release of FITC-insulin from the NanoCliP gel in PBS supplemented with $10 \%$ FBS at $37^{\circ} \mathrm{C}$. Results are expressed as the mean \pm standard deviation $(n=3)$. 
Figure 6 | Confocal LSM images of the NanoCliP gel following interactions with liposomes. (a) NanoCliP gel, (b) NBD-labeled liposome, (c) Superimposed image of a and b. Scale bar $=100 \mu \mathrm{m}$.

Figure 7 | Live-dead double-stained images of NIH3T3 cells adhered to the NanoCliP gel 24 h after cell seeding. (a) Live cells stained with calcein-AM. (b) Dead cells stained with propidium iodide. (c) Differential interference contrast image. (d) Superimposed image of a-c. (e, f) Depth distribution of live cells obtained by 3D confocal LSM. The insets show higher magnification images (white) and an F-actin-stained magnified image (red). Scale bar $=100 \mu \mathrm{m}$, inset, $20 \mu \mathrm{m}$.

Figure 8 | Histology of subcutaneously implanted NanoCliP gel in mice. (a) H\&E staining of the NanoCliP gel 4 weeks after transplantation. Arrows indicate the newly formed vessels in the NanoCliP gel. (b) The confocal LSM image of the rhodamine-labelled NanoCliP gel confirms that the NanoCliP gel has remained in situ. (c) Superimposed image of $\mathrm{a}$ and $\mathrm{b}$ shows the region containing the remaining NanoCliP gel. (D) F4/80 staining shows no monocytes and macrophages in or around the transplanted NanoCliP gel. Scale bar $=100 \mu \mathrm{m}$. 

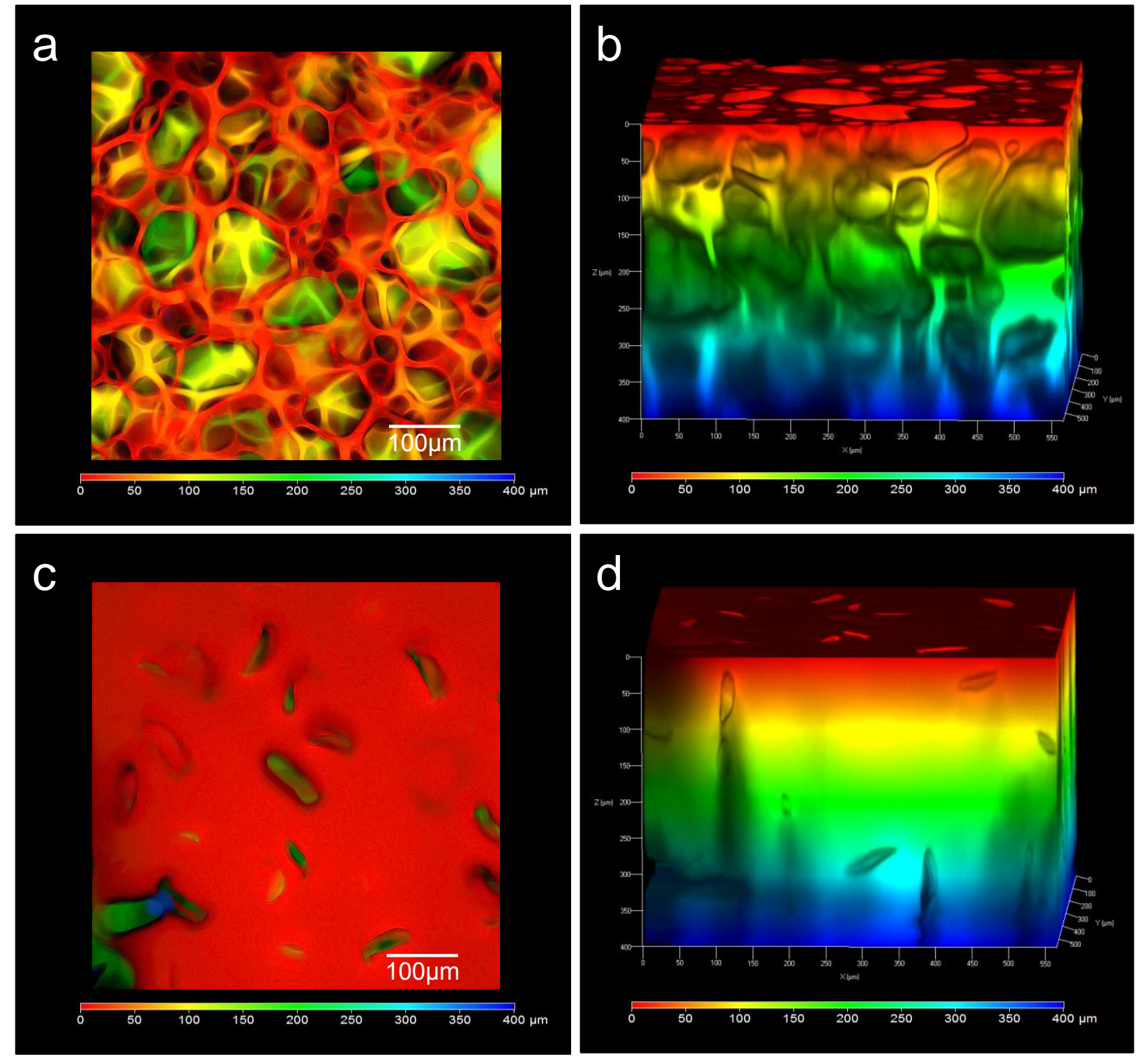

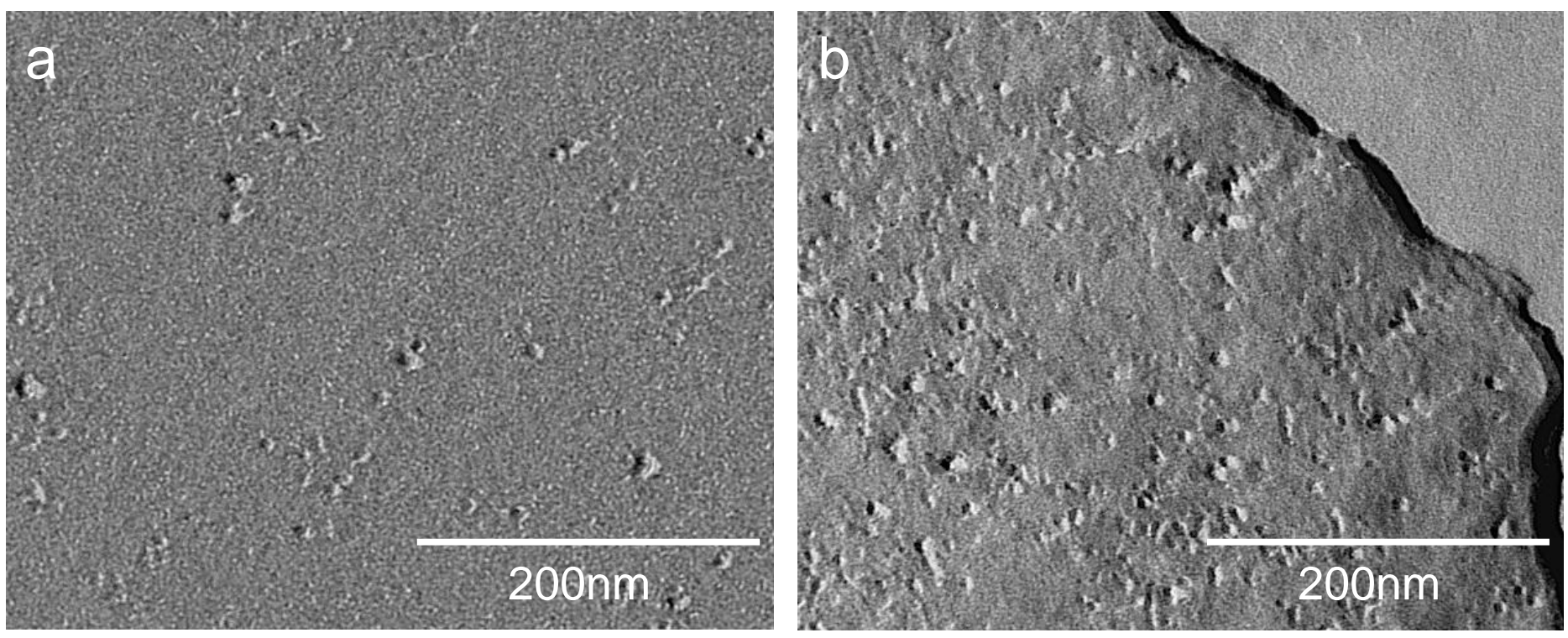
Figure 4 (Hashimoto et al.)
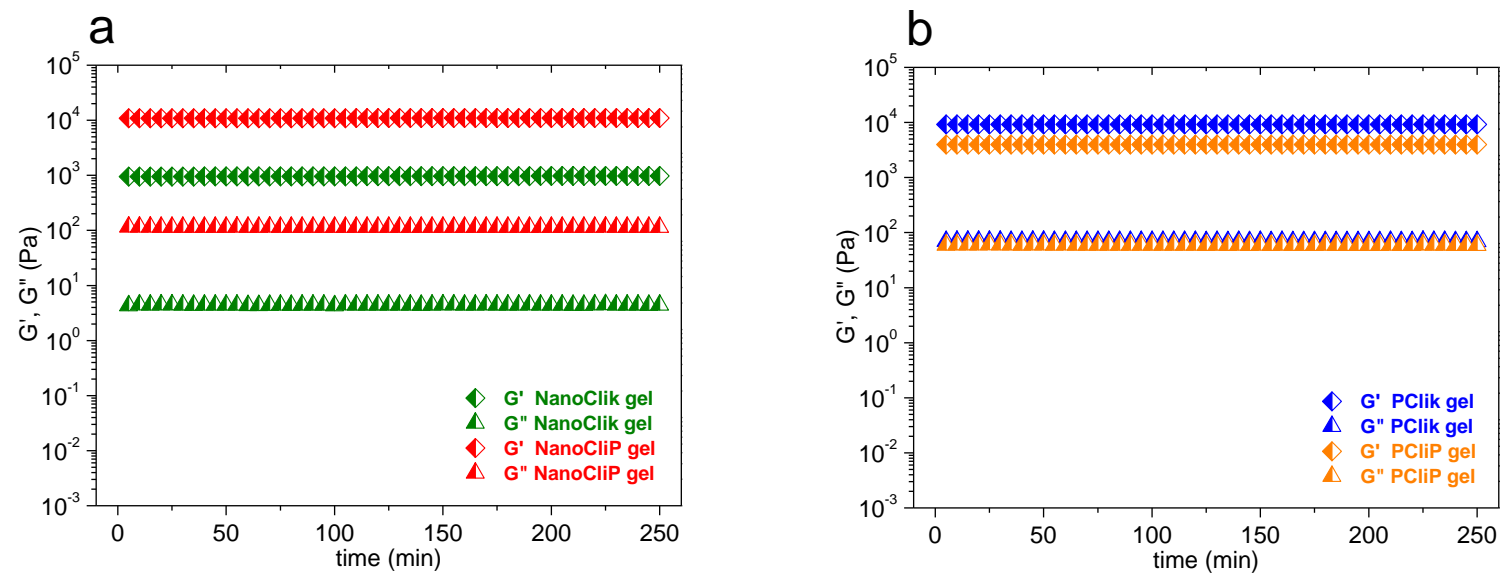

$$
\text { C }
$$
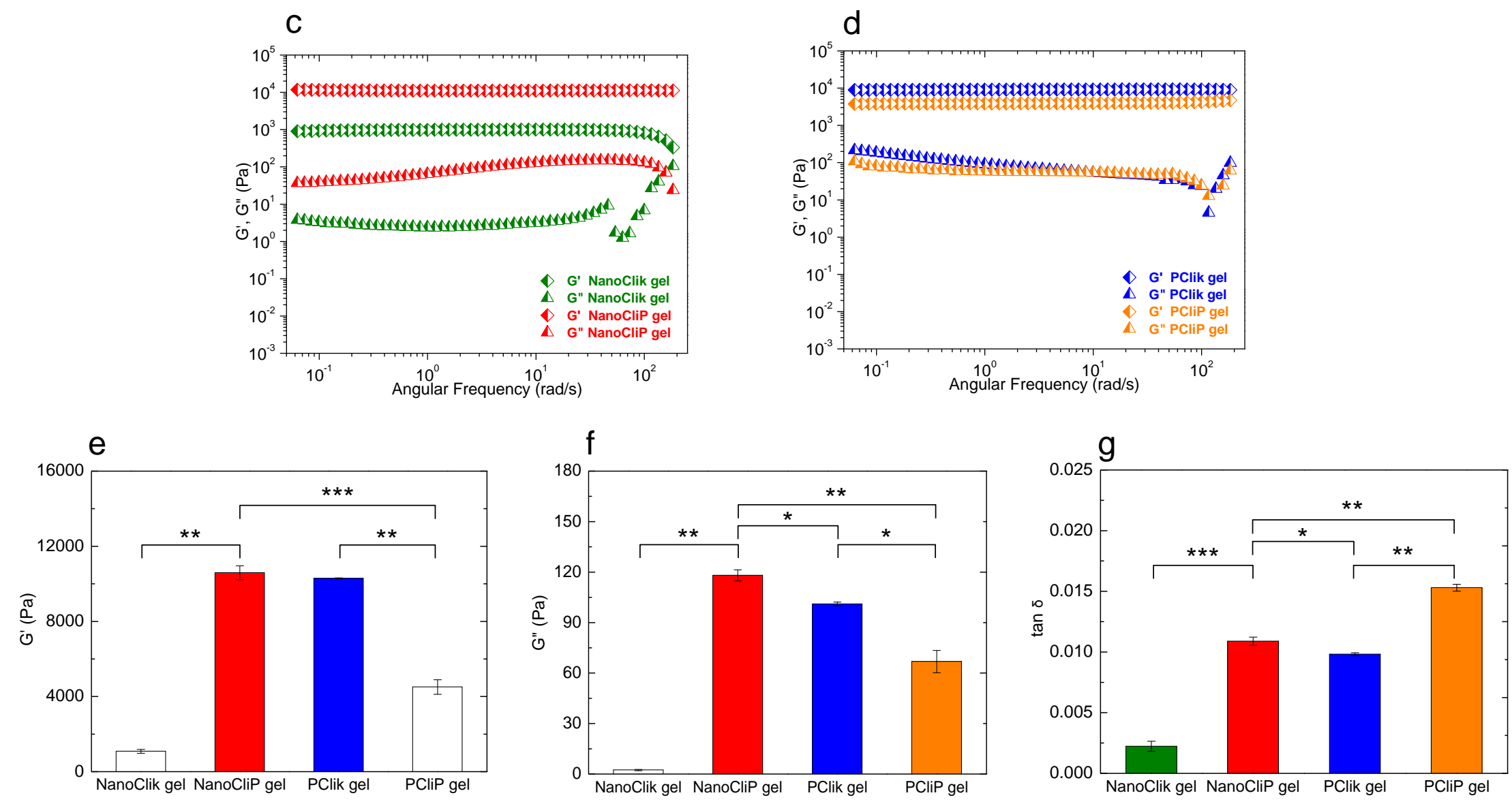

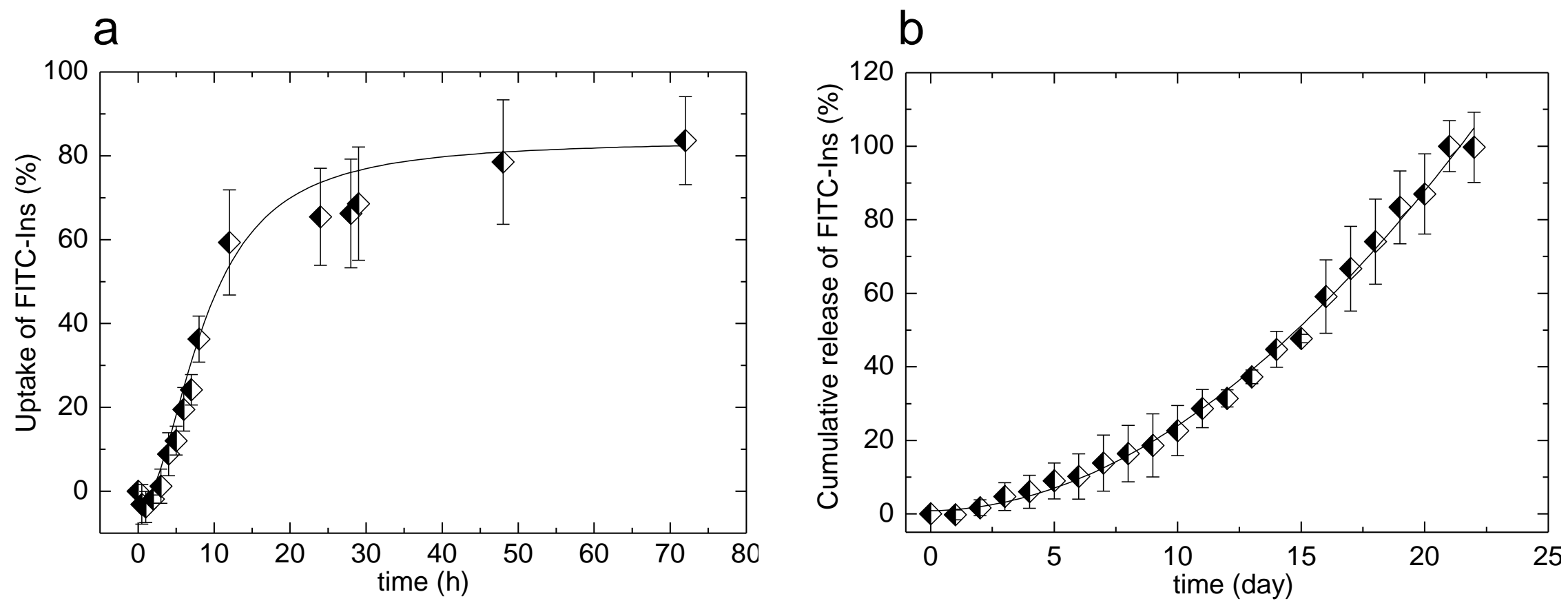

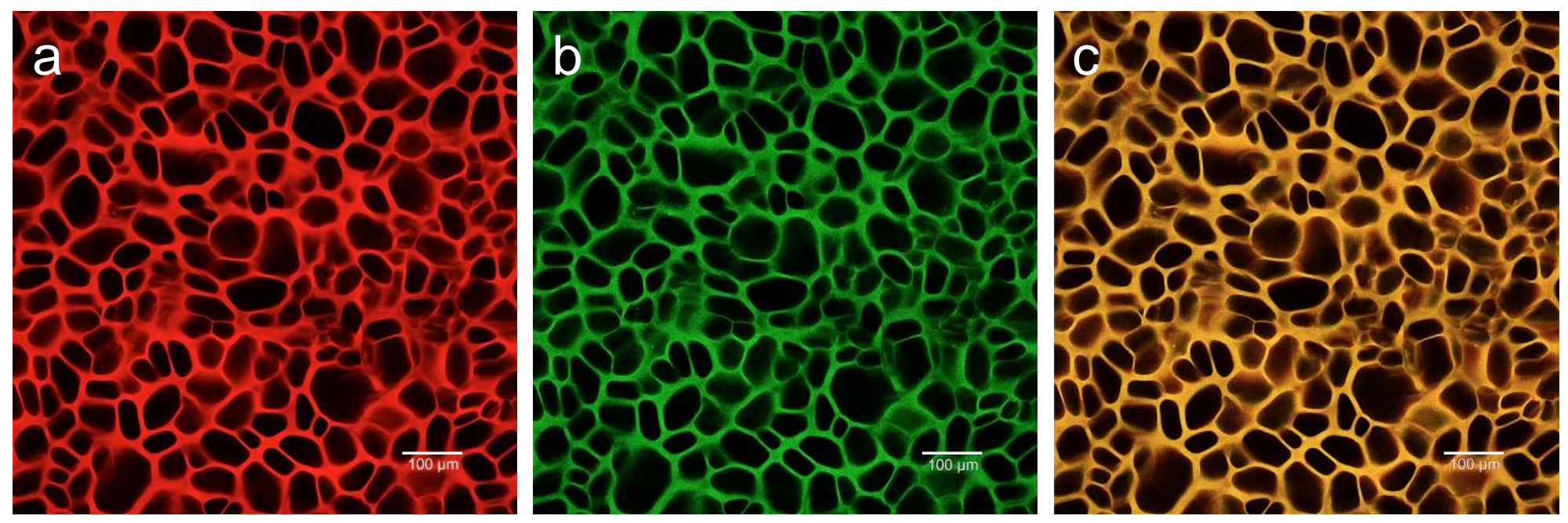

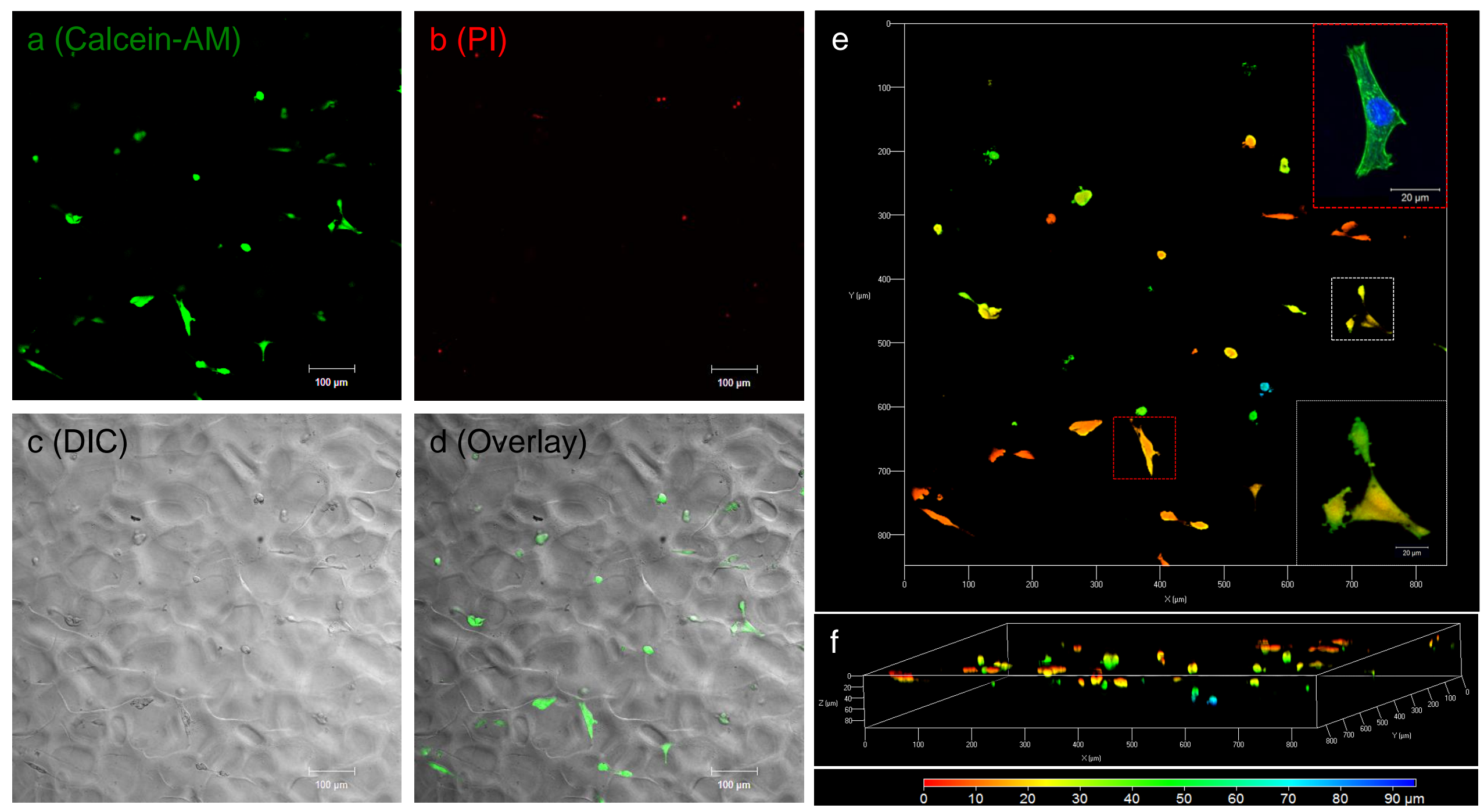

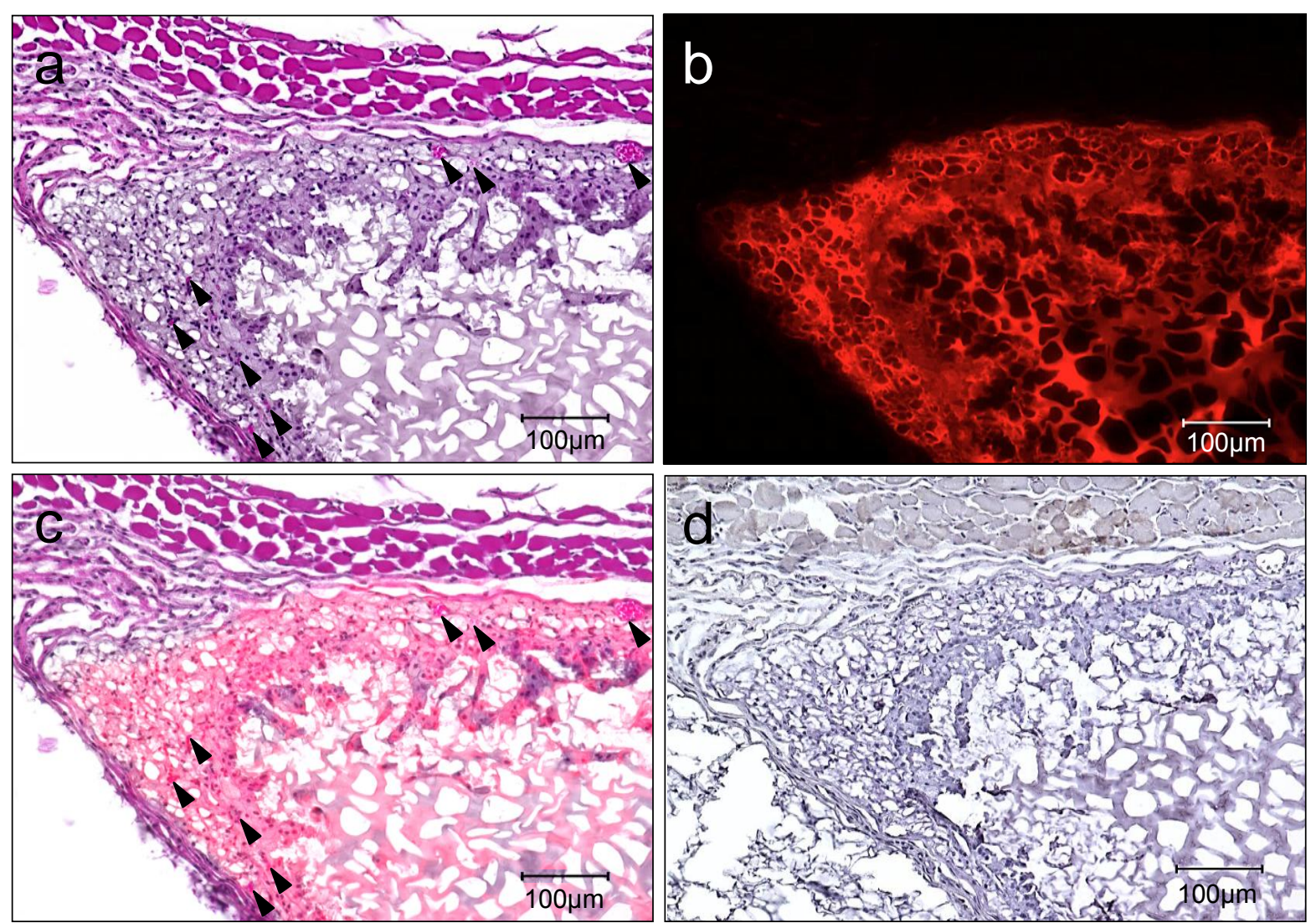